\title{
Clustering of Enterococcus faecalis Infections in a Cardiology Hospital Neonatal Intensive Care Unit
}

\author{
Tânia Mara Varejão Strabelli, \\ Daiane P. Cais, Rogério Zeigler, \\ Rinaldo Siciliano, Cristhieni Rodrigues, \\ Dirceu Carrara, Suzi Neres, Sarita Lessa \\ and David Everson Uip
}

\author{
Infection Control Unit, Heart Institute (InCor) of the \\ University of São Paulo Medical School; São Paulo, SP. Brazil
}

\begin{abstract}
Early identification of an outbreak is one of the main advantages of routine epidemiological surveillance. Enterococcus spp. used to be regarded as microorganisms of low pathogenicity, because they are part of the normal microbial flora of the gastrointestinal and genitourinary tract. Recently, they have emerged as important pathogenic agents, sometimes causing infections with high mortality rates. We studied a clustering of primary bloodstream infections caused by Enterococcus faecalis in a cardiology hospital neonatal intensive care unit (NICU). Four cases of primary bloodstream infection by $E$. faecalis were detected from April 15 to May 13, 2004, during active infection surveillance. The isolates were sensitive to glycopeptides. Some aspects of the management of these patients, including the date of insertion and placement of a central venous catheter, prescription of a specific medication, contiguity of beds, personnel attending the patients, and occurrence of diarrhea were analyzed to look for factors that might affect the spread of the microorganisms. Measures taken to hamper the spread included contact precautions throughout the unit, cleansing and disinfection of equipment and surfaces, bathing children with $2 \%$ chlorhexidine-gluconate-containing soap, professional reeducation, and reinforcement of all measures to prevent infections. We suggest that there is a need to re-evaluate preventive infection measures and to review the strategies aimed at decreasing the nosocomial infection rate in the NICU.
\end{abstract}

Key Words: Enterococcus faecalis, clustering, neonatal intensive care unit, bloodstream infection.

Enterococci are Gram-positive facultatively-aerobic, primarily-saprophytic bacteria living in the intestinal tract of animals, including humans. Recently, they have emerged as important pathogenic agents in nosocomial environments; they can cause severe infection with high mortality rates. From 1986 to 1989 , they were the second-most commonly isolated agent of hospital-acquired infections in the United States [1,2]. They can survive over long periods on inanimate objects such as thermometers and stethoscopes, and also on the hands of healthcare personnel; they often colonize the gastrointestinal tract of patients and healthcare workers as well. Outbreaks and endemic infections caused by Enterococcus spp. are an indication of patient-to-patient transmission [3], though the role of the environment in transmission is not clear [2].

Because of the selective pressure posed by the overuse of antibiotics in nosocomial environments, Enterococcus spp. have markedly increased in frequency as etiological agents of urinary tract and bloodstream infections (BSI). Furthermore, the severity of the patients' underlying condition, long-term

Received on 15 October 2005; revised 03 March 2006.

Address for correspondence: Dr. Tânia Mara V. Strabelli. Rua Voluntários da Pátria, 2831 apto 151, São Paulo, São Paulo, Brazil. ZIP 02401 100. Telephone No: +55-11-30695358. Fax: +55-11-30695349. E-mail: tania.strabelli@incor.usp.br

The Brazilian Journal of Infectious Diseases 2006;10(2):113-116. (C) 2006 by The Brazilian Journal of Infectious Diseases and Contexto Publishing. All rights reserved. in-hospital internment, prior surgery, renal insufficiency, presence of invasive devices, and long stays in the intensive care unit (ICU), are all additional risk factors for the occurrence of hospital-acquired infections [1].

From April 15 to May 13, 2004, four primary bloodstream infections by E. faecalis occurred in the cardiac neonatal intensive care unit (NICU) of a teaching hospital in the city of São Paulo, Brazil; no infection by this agent had been reported in the immediately-preceding 27-month period. An investigation was made to determine the source of the infections, and steps were taken to hamper transmission of this bacterium inside the unit.

\section{Material and Methods}

Setting (description of the unit and dynamics of attendance). The Heart Institute (InCor) of the University of São Paulo Medical School Hospital is a 490-bed tertiary teaching hospital specialized in cardiology, which predominantly treats surgical patients. The NICU has 10 beds, two of which are for patients who require expanded precautions. The patients are admitted from the emergency room, from hospital wards, from surgical ICU's or as referrals from other health-care services. Children admitted to the NICU are those with complex cardiopathy, and they frequently have to use medical devices, such as mechanical ventilation, central venous catheters and peritoneal dialysis.

At the time of the clustering of infection cases, the unit had a common entrance door coming straight from the nursing 
staff counter, with eight beds placed in two separate rooms. The entrance to the room with the two expanded precaution beds was through a lateral door with an entrance hall and a sink; communication of this room with the rest of the unit was through a glass door separating the internal part of the unit. This layout is displayed in Figure 1.

Figure 1. Layout of the neonatal intensive care unit during the clustering.

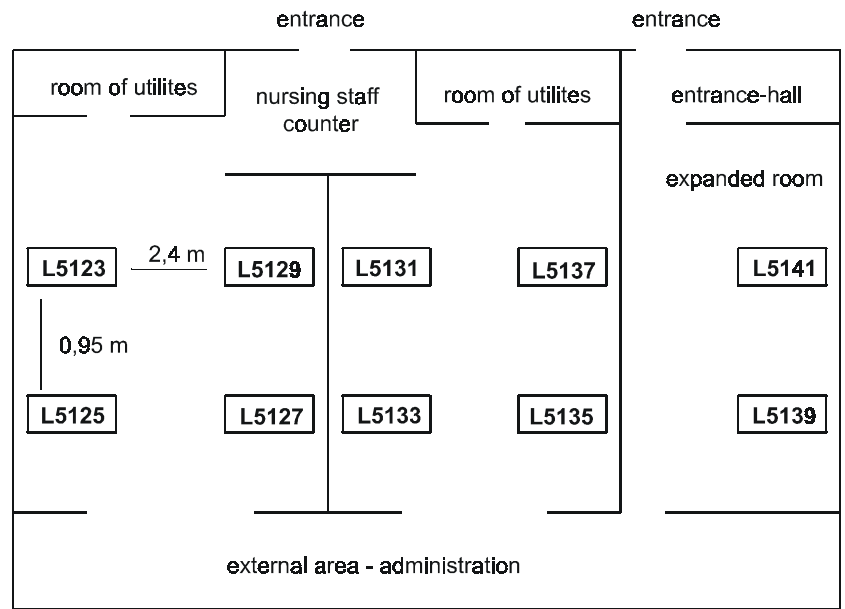

$\mathrm{L}=$ bed number.

During day shifts, the staff consisted of a physician and three medical students. The night shift was attended by a physician and two medical students. A physiotherapist and a trainee worked in the morning and afternoon shifts, whereas during the night shift a physiotherapist was on-call whenever needed, attending a maximum of two children in the unit. The patient/nurse ratio was 1.8 during workdays. During weekends, holidays and night shifts, the ratio was 2.25 patients per nurse.

Patient inclusion criteria. A study patient was defined as a child admitted to the unit at least 48 hours before, having at least one positive blood culture for E. faecalis, and this pathogen was not related to infection at another site [4].

Cultures. The blood samples were collected with aseptic technique by venipuncture $(2 \mathrm{~mL} / \mathrm{set})$, and they were analyzed at the Microbiology Laboratory, Central Lab Division, Department of Pathology, University of São Paulo Medical School (HCFMUSP). In the lab, the blood specimens were processed in a Bactec 9240 system (Becton Dickinson, Sparks, MD). After strain identification, antibiotic susceptibility testing was conducted according to recommendations of the National Committee for Clinical Laboratory Standards (NCCLS), using the Vitek ${ }^{\circledR}$ (BioMérieux/USA) card.

The Infection Control Unit received copies of every positive culture from all the patients admitted to the hospital
Epidemiological surveillance. Epidemiological surveillance was carried out by two nurses, both of whom worked eight hours a day. Active surveillance was carried out through daily visits to the unit, using the ICU component of the NNISS (National Nosocomial Infections Surveillance System) methodology, based on Centers for Disease Control and Prevention (CDC) guidelines [5].

All of the data were organized and analyzed with the dedicated software Doctor Clean, which enables analysis of data and periodic reports.

Measures to prevent hospital-acquired infections. Hand hygiene followed the hospital guidelines, which recommend an alcohol-based hand rub or the use of $2 \%$ chlorhexidinegluconate-containing soap in critical areas, before and after any patient contact [6].

The Hospital Infection Control Team has periodically revised hospital-acquired infections prevention guidelines, which can be accessed via intranet throughout the hospital. In addition, one nurse is in charge of continued education of the staff, through lectures and workshops, wherever routine techniques are carried out.

A program to restrict access to broad-spectrum antibiotics has existed since 1987. It is in-use during seven days a week, around the clock, by infectious disease doctors and pharmacy staff.

\section{Results}

Four patients presented with bloodstream infection by $E$. faecalis; this bacterium was not isolated from any other site from April 15 to May 13, 2004. No infection by this agent was reported in the previous 27 -month period.

Twenty-eight children were admitted to the ICU during the study period. The occupancy rate was $96 \%$; all were children with highly complex clinical conditions. Although the expanded precaution room had two beds, only one of them was occupied during the study. The number of patientdays was 251 and catheter-days was 241 . The rate of device utilization (DU) was $249 \%$ (68\% indwelling vesical catheters, $85 \%$ mechanical ventilation support, and $96 \%$ central venous catheters).

The four patients are described in detail below.

Patient 1. KCFS, 16-month-old, female, 13th postoperative day after mitral valve repair surgery. A positive blood culture was detected April 15, diagnosed as a bloodstream infection (BSI) by E. faecalis, which was sensitive to aminoglycosides and glycopeptides. At this time, the child was in septic shock and was undergoing peritoneal dialysis with a Tenckhoff catheter. She received vancomycin (18 days) and meropenem (15 days); the E. faecalis BSI was treated with ampicillin and gentamicin. 
Patient 2. BRS, 16 month-old, female, 40th postoperative day after correction of aorta coarctation, surgical treatment of ductus arteriosus and lung biopsy. A positive blood culture was identified on April 23 (E. faecalis resistant to gentamicin and Pseudomonas paucimobilis). At this time, this child had a fever ( $38^{\circ} \mathrm{C}$ axillary), 13,600 leukocytes $/ \mathrm{mm}^{3}$, was undergoing peritoneal dialysis with a Tenckhoff catheter, and had normal blood pressure. She received piperacillin/tazobactam (12 days) and Amphotericin (18 days); polymixyn B was introduced to treat an Acinetobacter baumannii endocarditis. Enterococcus faecalis BSI was treated with vancomycin. The patient died on May 15.

Patient 3. MVFS, seven month-old, male, 25th postoperative day after a Blalock-Taussig operation. This patient shared the room with another patient identified as positive for rotavirus and had diarrhea from April 27 to 30. On May 12, a BSI was identified (blood and central catheter tip culture positive for Acinetobacter baumannii and E. faecalis sensitive to aminoglycosides and glycopeptides). At this time, the child had a fever (38. $1^{\circ} \mathrm{C}$ axillary), arterial hypotension, and 19,400 leukocytes $/ \mathrm{mm}^{3}$. He received vancomycin (two days) and cefepime (two days). He died on June 8.

Patient 4. GDP, three month-old male, seventh postoperative day after a pulmonary trunk bandage and surgical treatment of ductus arteriosus. He had fever, 14,500 leukocytes $/ \mathrm{mm}^{3}$ and was not receiving antibiotics. After BSI diagnosis, he was treated with vancomycin, ampicillin and gentamicin. He was discharged from the hospital on August 12.

Figure 2 displays the length of time of use of the central venous catheter, as well as the date when BSI was diagnosed and the diarrhea episodes in the patients. No health care worker reported diarrhea episodes during the clustering.

Figure 2. Description of length of time of use of the central venous catheter, diarrhea episodes and positive blood cultures for the four patients infected by Enterococcus faecalis

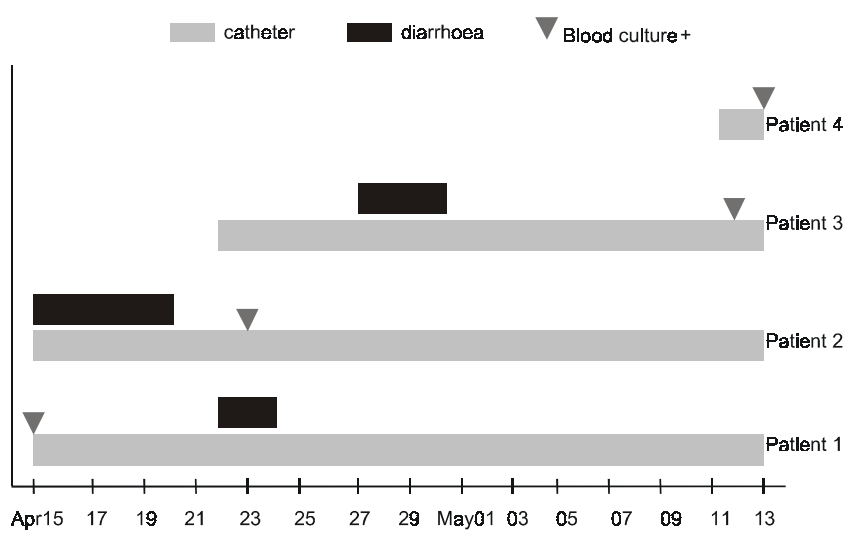

All children had a central venous catheter during this period; none of them had a femoral access that could possibly be correlated with the occurrence of diarrhea.

The medication prescribed for the patients was the usual for this hospital, which goes against the hypothesis of occurrence of contaminated fluids infusion.

Patients 1 and 2 occupied adjacent beds for 12 days before the clustering was diagnosed.

Measures implemented after the outbreak was identified. The following steps were taken to hamper dissemination of $E$. faecalis:

1. Interruption of new patients admissions to the unit and of discharges from it during one week, so as to modify the unit layout. The entrance through the nursing staff counter was closed. An ordinary room was modified to serve as the unit entrance, as shown in Figure 3.

2 . Bathing of all children with $2 \%$ chlorhexidine-gluconatecontaining soap, after the clustering was identified.

3. Cleansing of all furniture and equipment in the unit with water and neutral soap, and $70 \%$ ethanol solution disinfection. Wood floor planks were replaced by planks made of acrylic resin, which were disinfected with $70 \%$ alcohol once a week thereafter.

4. Re-sizing of the nursing staff: admission of five healthcare workers $(\mathrm{HCW})$ to fill vacancies due to dismissals and licenses.

5. Informing all $\mathrm{HCW}$ about the clustering, reinforcing the importance of their adhesion to hospital-acquired infection prevention recommendations.

\section{Discussion}

We found that active surveillance through continuous registration of hospital infections and efficient registry of microbiological data are vital to permit early identification and investigation of clustering in any hospital. This monitoring is even more important in ICUs, especially NICUs, due to the patients' immunosuppression caused by their underlying conditions and/or due to therapeutic procedures [2]. All children admitted to this unit had at least one central venous catheter, which broke the skin barrier, rendering them susceptible to direct contact between their bloodstream and the environment [7].

Unfortunately, the enterococci strains were not typed, and no surveillance cultures were made to determine carrier status. So there was no conclusive evidence to support the possibility that this event was an outbreak.

No common source of infection was detected during this clustering. There was a temporal relationship between BSI by E. faecalis and the occurrence of episodes of Rotavirus diarrhea in the NICU. This may have increased the exposition to faeces colonized by enterococci, associated with increased catheter use by these patients. The replacement of wood floor 
Figure 3. Layout of the neonatal intensive care unit after the clustering

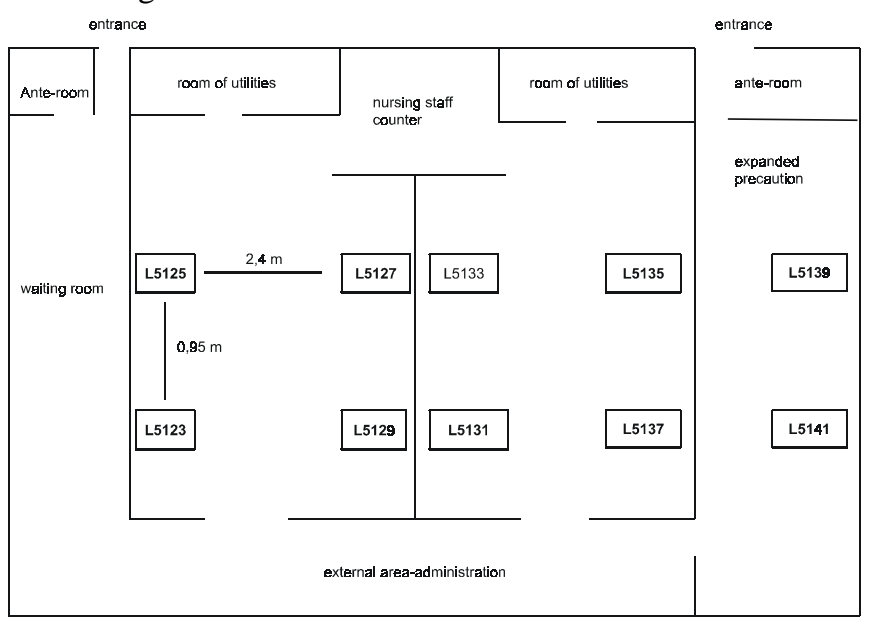

planks by others that were easier to clean (acrylic resin) was a timely measure $[8,9]$.

In our study, the dissemination of E. faecalis may be attributed to the excessive workload (severity of the patients' conditions associated with understaffing), which reduced adhesion to basic preventive measures needed to avoid hospital-acquired infection. Among others, some risk factors that may be related to this clustering were a high rate of bed occupancy and a long stay in the unit; both of these predisposing factors were also reported by Andersen et al. [9].

Other studies have reported spread of infections directly related to understaffing and excessive workload [2,9-11]. Harbarth et al. concluded that understaffing during periods of increased workload can result in additional nosocomial infections and costs, which are surrogate markers of poor quality of care [11].

Another concern about Enterococcus spp. is its ever-increasing resistance to antibiotics [2]. Excessive usage of antimicrobial agents is a risk factor for increased resistance, colonization and infection by enterococci. This is the reason why hospitals must have a solid program of antibiotic-usage control $[8,12]$.

Samuelsson et al. reported an increase in the number of BSI by E. faecalis in ICUs, mentioning that, among many other steps to hamper such transmission, one should change the organization of beds and increase the distance between patients [2]. Layout modification, in our study, did not afford any significant increase in distance between beds; however restricting the flow to the nursing staff counter may have been one of the factors that contributed towards halting dissemination of the pathogenic agents.

In conclusion, epidemiological surveillance and a combination of all measures implemented in the unit were important to identify and stop the spread at an early stage $[2,8,9]$. During the ensuing semester we did not observe any other case of primary bloodstream infection by E. faecalis.

\section{References}

1. Fernandes A.T., Ribeiro Filho N., Mazzano R.S., et al. Bactérias aeróbias. In: Fernandes A.T., Fernandes M.A.V., Ribeiro Filho N. Infecção hospitalar e suas interfaces na área de saúde. (Hospital-acquired infection and its interfaces in the area of healthcare). São Paulo: Atheneu, 2000;14:345-6.

2. Samuelsson A., Jonasson J., Mosntein H.-J., et al. Clustering of enterococcal infections in a general intensive care unit. J Hosp Infection 2003; 54 : 188-95.

3. Takahashi S., Hirose T., Matsukawa M., et al. Analysis of cross infection using genomic fingerprinting in nosocomial urinary tract infection caused by Enterococcus faecalis. J Infect Chemother 1999;5:46-8.

4. Centers for Disease Control and Prevention. Guidelines for the Prevention of Intravascular Catheter-Related Infections. MMWR 2002; 51(RR-10):1-36

5. Centers for Disease Control and Prevention. NNIS Manual. US Department of Health and Human Services, Atlanta, Georgia: 1992.

6. Boyce JM, Pittet D; Healthcare Infection Control Practices Advisory Committee. Society for Healthcare Epidemiology of America. Association for Professionals in Infection Control. Infectious Diseases Society of America. Hand Hygiene Task Force. Guideline for Hand Hygiene in Health-Care Settings: recommendations of the Healthcare Infection Control Practices Advisory Committee and the HICPAC/SHEA/APIC/IDSA Hand Hygiene Task Force. Infect Control Hosp Epidemiol 2002;23(12 Suppl):S3-40.

7. Pittet D., Hervaldt L.A., Massari R.M. The intensive care unit. In: Bennett J.V. \& Brachman P.S. Hospital infections. Boston: Little Brown, $3^{\text {a }}$ ed., 1992;20:405-39.

8. Associação Paulista de Estudos e Controle de Infecção Hospitalar. Enterococos resistente aos glicopeptídeos. (Glycopeptideresistant enterococci). São Paulo, 1999.

9. Andersen B.M., Lindermann R., Bergh K., et al. Spread of methicillin-resistant Staphylococcus aureus in a neonatal intensive unit associated with understaffing, overcrowding and mixing of patients. J Hosp Infection 2002;50:18-24.

10. Roberts S.A., Findlay R., Lang S.D.R. Investigation of an outbreak of multi-drug resistant Acinetobacter baumannii in an intensive care burns unit. J Hosp Infection 2001;48:22832.

11. Harbarth S., Sudre P., Dharan S., et al. Outbreak of Enterobacter cloacae related to understaffing, overcrowding, and poor hygiene practices. Infect Control Hosp Epidemiol 1999;20:598-603.

12. Associação Paulista de Estudos e Controle de Infecção Hospitalar. Melhorando o uso de antimicrobianos em hospitais (Improving usage of antimicrobial agents in hospitals) São Paulo, 2002, p.4. 